\title{
A New Approach Based on Order Reduction Using Sub Image Formation in Minimizing the Computation Time for Image Compression
}

\author{
${ }^{1}$ Shaik, Mahaboob Basha and ${ }^{2}$ B. C. Jinaga \\ ${ }^{1}$ Professor and Head of the Department of E.C.E, Priyadarshini College of \\ Engineering and Technology, Nellore, Andhra Pradesh, India, \\ ${ }^{2}$ Professor in E.C.E (Retd), Department of E.C.E, J.N.T.University, Hyderabad, \\ Telangana., India \\ ${ }^{1}$ mohisin7@yahoo.co.in, ${ }^{2}$ jinagabc@yahoo.co.in
}

\begin{abstract}
Image compression is the process to remove the redundant information from the image so that only essential information can be stored to reduce the storage size and transmission time. Advancement in still image compression becomes essential for various applications like medical imaging and multimedia applications. The task of image compression generally refers to deriving a near approximated compressed image for the given input image. Methods for tackling this problem have to do a delicate balancing act of suppressing the unwanted effects in visualizing without losing features of interest.

Different techniques were already proposed in this area which is not sufficient to maintain good quality of the compressed image without sacrificing the compression ratio. Thus, new image compression algorithm based on order reduction using sub-image formation is proposed for lossy compression scheme. Obviously, the tradeoff between compression ratio and picture quality is an important issue in image compression. The importance of running time of compression was further investigated as this process can be used in high speed data transmission applications. The simulation process is carried out determining the computation time using MATLAB. The algorithm tested for still images of different size too.
\end{abstract}

Keywords: image compression, order reduction,lossy compression, computation time, sub-image

\section{Introduction}

The fast moving electronic industry has made it inevitable for the modern man to move with the images it presented through various means like video and web cameras. These images are functioning on standard algorithms were commonly inducted for all image processing applications. Thus, the research calls for the mathematical techniques, often them the standard algorithms to solve the forward and inverse problems. The solution reached and lead to a novel mathematical approach, the order reduction based compression technique for lossy still image compression, which is the prime of this work.

An image may consist of thousands to millions of pixels. The compression process reduces the unwanted redundant information and implies to minimize the computation time. At the same time, the magnitude of the compression ratio also should not be neglected. Iterative schemes with algorithms were tried to have clean visuals after reconstruction of the 
image without sacrificing any information. Some of the resultant reduced model was close and nearer to the originals. This was the motivating factor for the formulation of the novel idea for the evaluation of a lossy compression algorithm.

\section{Literature Survey}

A lossy compression technique was observed for deducing higher compression efficiency and PSNR values calculated in maximum is $37.770 \mathrm{~dB}$ in [1]. Another technique was introduced as MODIS data for Image compression in [2] and the values of PSNR for JPEG, SPIHT and JPEG 2000. A novel Context based Binary Wavelet Transform Coding approach (CBWTC) that combines the BWT with a high order context based arithmetic coding scheme to embedded compression of grayscale images in [3]. In [4] two compression methods were presented for irregular 3D mesh sequences with constant connectivity by using an exact integer spatial wavelet analysis (SWA) technique.

Yi Zhang and Xingyuan Wang [5] proposed a fractal image compression coding scheme based on wavelet transform with diamond search. In [6] a novel technique was proposed a new image coding method based on discrete directional wavelet transform (S-WT) and quad tree decomposition. A new linked significant tree (LST) wavelet coding method for improved compression of images together within a wavelet tree to facilitate encoding algorithm was seen in [7]. In [8], we observed a new wavelet transform image coding algorithm with the discrete wavelet transform (DWT) is applied on the original image. Isa Servan Uzun and Abbes Amira [9] reported the design and field programmable gate array (FPGA) implementation of a non-separable compression system.

Two categories of transform coding and subband coding and for compressing ultrasonic NDE images were studied in [10]. The [11] proposed a new stereo image compression scheme that is based on the wavelet transform of both images and the disparity estimation between the stereo pair sub bands. The [12] presented a technique for MR image compression based on a transform coding scheme using the wavelet transform and vector quantization.

Elisabetta Delponte et al. [6] proposed the continuation work of SVD Matching of Scott and Longest Higgins and Pilu and proposed new technique using the features of SIFT. The Proximity matrix was removed in the work.

The analysis of the image processing with multidimensional modeling techniques and derivation of the static nature in the images, a wonderful approach to attract the researchers towards the comprehensive knowledge on mathematics was highlighted in [13]. Pamela C. Cosman et al. [19] derived an approach for estimating the SNR and the visual appearance of the compressed medical images.

The image distortion and quality measures are simultaneously. The pure orientation filtering for image processing was used is invariant for scales. The Invariance property is applicable for image processing and signal processing also. Biological and perception applications were discussed in detail in [15].

\section{Proposed Order Reduction Technique based on Sub-image Formation}

An image is consisting of thousands of pixels. The process reduces the unwanted redundant information to reduce the computation time by increasing the compression ratio. Iterative schemes with algorithms where tried to have clean visuals after reconstruction of the image without sacrificing any information. Some of the resultant reduced model was 
close and nearer to the originals. This was the motivating factor for the formulation of the novel ideas for the research done on lossy and lossless compression algorithms.

\subsection{Static Sub-image Concept}

Image compression is one of the most important applications of digital image processing. Advanced medical imaging requires storing large quantities of digitized clinical data. In this technique, static sub-images are to be constructed from the selected input image. For each sub-image the matrixes are categorized. All the sub-images are assembled to formulate the compressed or reduced model of the complete structure. The compressed image is usually much smaller than the input bench mark image. The proposed algorithm which is based on the order reduction technique is a new paradigm in image compression. Static image exhibits the time and spatial based property. General still images are static images.

The first computation in the proposed algorithm is the generation of the sub image which has diagonal form. This matrix as per requirement of the order reduction methodology adapted here has to posses dominant features in discrete sense. This is strict constraint for achieving perfect reconstruction. To be considered as a static sub-image, the group of elements must meet certain mathematical conditions [23]. The algorithm can be categorized into three parts. The initial stage involves the input image is being decomposed to required number of sub-images. The sub-images may exhibit different mathematical geometric properties. Here the task of selecting one sub image from the set of images is important, as this selected sub image features are solely responsible for reconstruction. The order reduction technique plays the role of this selection, where the degrees of freedom can be chosen by the user. The order reduction technique uses the appropriate decomposition which consumes less computation, as the matrix computation of higher order always are tedious processing risks requiring huge computation time. The proposed algorithm is developed by making use of LU decomposition for achieving less computation time. The size of the reduced image obtained from this technique is usually much smaller than that of the original image.

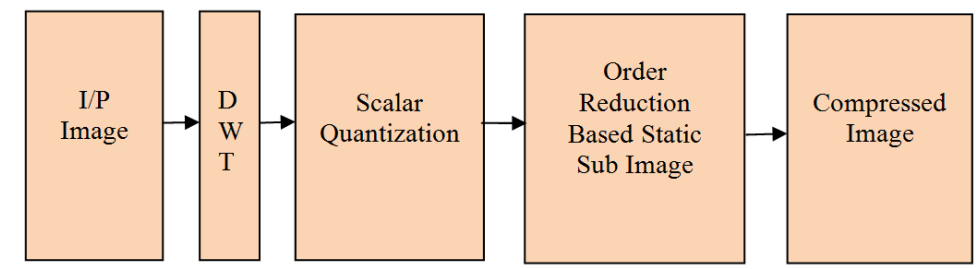

Figure 1. Block Diagram of Static Sub-image Technique Compression

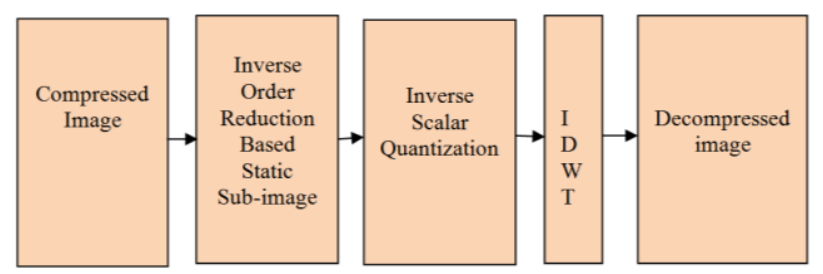

Figure 2. Block Diagram of Static Sub-image Technique Decompression 


\subsection{Advantages of the Static Sub-image Technique}

1. The sub-image construction is easily formulated with the order reduction methods.

2. The static sub-image generated is a very effective projection matrix that is used for desired compression.

The block diagram of the proposed algorithm is shown in figures 1 and 2 .

\subsubsection{Wavelet Transform}

The basics and importance of the usage of wavelets explained in the following section. Wavelets are small signal approximations. They have become very popular in 1-D signal processing, 2-D image processing and 3-D image processing applications. They are defined mathematically within a finite interval time. There are many wavelets existing, and can also be constructed from our own intuition. But they should satisfy certain properties like having average value of zero [24].

Wavelet transform begins with choosing proper mother wavelet. From the chosen basis, sub wavelet families are obtained by dilations or contractions and translations. Dilations refer the scaling operation and translations refer the shifting operation. These have found usage in signal and image processing, where the time frequency resolution is requires for analysis. They emerged as substitution to Fourier transform for many real time applications. Starting from the image compression of fingerprint images wavelets also found place in radar applications, detecting earthquakes, ECG, EEG analysis to name few. Different transforms exhibit different properties in spatial and statistical domains. Hence it was chosen the required wavelet for the appropriate application. The higher scales have stretched wavelets. The wavelet stretched more makes it possible to compare with long time duration signals, compressed wavelet provide us the coarse details.

\subsubsection{Wavelet Transforms of Image Signals}

The wavelet transform opened a new means of dealing image processing activities. They found their place in compression, watermarking, segmentation etc., very effectively. It results in decomposing an image in different approaches like for general sub band and packet transform. The outcome of the above said decomposition is emergence of four images in the form of matrices, with the dimensions of all the images being exactly half of the original image. The four matrices are obtained by means of four different approaches of dealing the row and column processing. The matrix which is processed in row and column with low pass filter banks is of interest, as it is called the approximated version. Above said approximated image can be further processed of yet another approximation, and the iteration is best stopped at the desired level of our interest.

\subsubsection{Discrete Wavelet Transform}

DWT has several families out of which, the Daubechies (Db4) with 9/7 tap filter is selected Lossy compression. Because of their compact support the Daubechies exhibit good support for image compression. They are very much part of the JPEG 2000 standard.

The LL pair of DWT decomposition is obtained by decomposing the given image in a column with the 9/7 filters coefficients of Daubechies Db4. This LL pair of a given $n x n$ image belonging to $k^{n+n}$ is decomposed to, $m x m$ image where $\mathrm{m}=\mathrm{n} / 2$, and $R$ now is changed in dimensions $R^{m+n}$..$L L$ band is the approximation coefficient of a given image, which has the low frequency components of pixel intensities. These pixel intensities of LL pair has 
most of the pixel variations needed for the image representation, where as $\mathrm{HH}$ pair has the high frequency components of an image for pressuring the edge. Hence HH, HL, LH bands of a given image are retained for reconstruction purpose. Hence, the processing of an image is primarily done on the LL band, i.e., the model order reduction is applied on the LL band in this case. The model order reduced version of the LL decomposed image is quantified in reconstruction process for by considering the image quality as reference.

\subsubsection{Scalar Quantization}

Scalar Quantization is necessary to arrange the DWT coefficients in prescribed set of amplitude levels. The quantization used can be either uniform or non uniform, but generally non-uniform for lossy image compression techniques. Non Uniform quantization varies one static sub-band image to another based on the visual models. The static image is decompressed by means of wavelet based MRA at level-2.

\subsubsection{LU Decomposition}

LU decomposition is a powerful method in solving large linear system matrices. In the process of getting higher compression efficiency and less computation times, the LU factorization was adopted. Initially, the DWT is applied on the input image and the image matrix is processed through LU factorization. $\mathrm{L}$ is lower triangle matrix and $\mathrm{U}$ is upper triangle matrix. This factorization method was considered because of its salient feature of predominant nature in inverting a matrix and to evaluate the matrix determinant factor.

\subsection{Static Sub-image Algorithm}

The static sub image based compression algorithm is as follows

Step1: The input image is considered with zero blur.

Step2: The input image is sub divided into sub images and estimated for number of pixels.

Step3: The divided sub images are made into diagonal matrices as U1, and V1.

Step4: LU Decomposition is processed and singular values of

$\mathrm{L}$ and $\mathrm{U}$ are computed and termed as $\mathrm{P}$.

Step5: The diagonal matrices of compressed image are estimated.

$$
L^{O}=U_{1} V_{1} \sum_{i=0}^{n} P_{i}
$$

$U_{l}$ and $V_{l}$ are diagonal matrices. $L^{0}$ is the compressed diagonal matrix.

\subsubsection{Iterative Scheme}

The step-wise sequence of the proposed iterative scheme was derived which in turn confirmed visual quality as well as compression ratio and computation times in the following section.

1. Partition the Chrominance and Luminance matrices and construct the corresponding sub matrices.

2. Factorize the matrices of the output LU Decomposition

3. Compute the initial approximation of the compressed (reduced) model is computed

4. Solve for the eigen values of the compressed (reduced) model.

5. Compute the Projection matrix

6. For $i=k, 2 k, \ldots(k \geq 1)$, begin the iteration: 
6.1. Compute the $(i)^{t h}$ approximation compression Matrix of LU decomposition

6.2. Calculate the $(i)^{t h}$ approximation of the order reduced matrices.

6.3. Solve for the reduced eigen values

6.4. Check for convergence where is a prescribed error tolerance and $m$ is a fixed value and are the $(i-1)^{\text {th }}$ and $(i)^{\text {th }}$ approximations values of the $(j)^{\text {th }}$ frequency of the compressed (reduced) model.

7. Iteration converges at good quality of the processed image.

8. Iteration loop exit.

During the iteration process, it was found that the quality of the compressed image had achieved with less number of iterations and the reduction in the computation time.

\section{Flow Chart Description}

In Static sub image based image compression, the input test image is first decomposed using Discrete Wavelet Transform. The input image is considered to be static in the sense that it is free from the blur.

The LL band is considered for the scalar quantization. The reason of choosing the LU decomposition is justified from the theme of the static sub image based compression where the compression is to be done in quick time.

The flow chart for the static sub image is shown in the Figure 3.The eigen values obtained from the LU decomposition are used in the iteration scheme of the algorithm as the initial values. The main idea of the proposed static sub image based compression algorithm is to have the dominant eigen values to be retained from the LL band to attain reduction in the computation time.

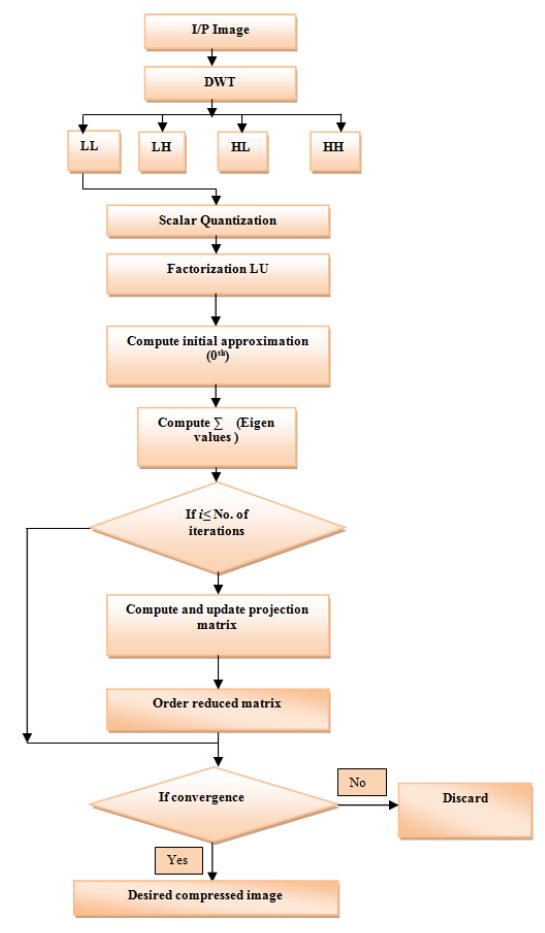

Figure 3. The Flow Chart of the Proposed Approach 


\section{Simulation Results}

The proposed Novel algorithm was implemented on several wide varieties of images. The detailed illustration of the simulations along with the output images and computation times are given in the following section. The comparison of existing techniques with the proposed is shown in the tables, and finally the comparison chart showcases the performance measure.

The test images have a size of $256 * 256$ colour and gray scale images. With these three images, the proposed method behaves better at a high compression rate. The compression matrix generated is in reference to the number of orientations. In any case, it gives better results using static sub image technique. The results clearly show the increase in the compression efficiency has reduced the CPU times in image compression. The computation values of the major contributions in this area are mentioned in the Table I. From these observations it is understood that the static sub-image technique surpasses the existing techniques.

The execution of static sub image technique based compression was carried out on different images including natural and medical. The proposed work is a twofold implementation, i.e., better compression and reducing the same time computation time. The original, compressed and decompressed images are shown in the figures mentioned in the following section.

\subsection{Chest Image}

The proposed work was also extended to investigate the performance of the algorithm for medical image processing, as medical images are also prone to computation time constraint seriously. The chest image was selected from the set of medical images as our proposed work has found to be fit for it in minimizing the computation time while compressing the image.

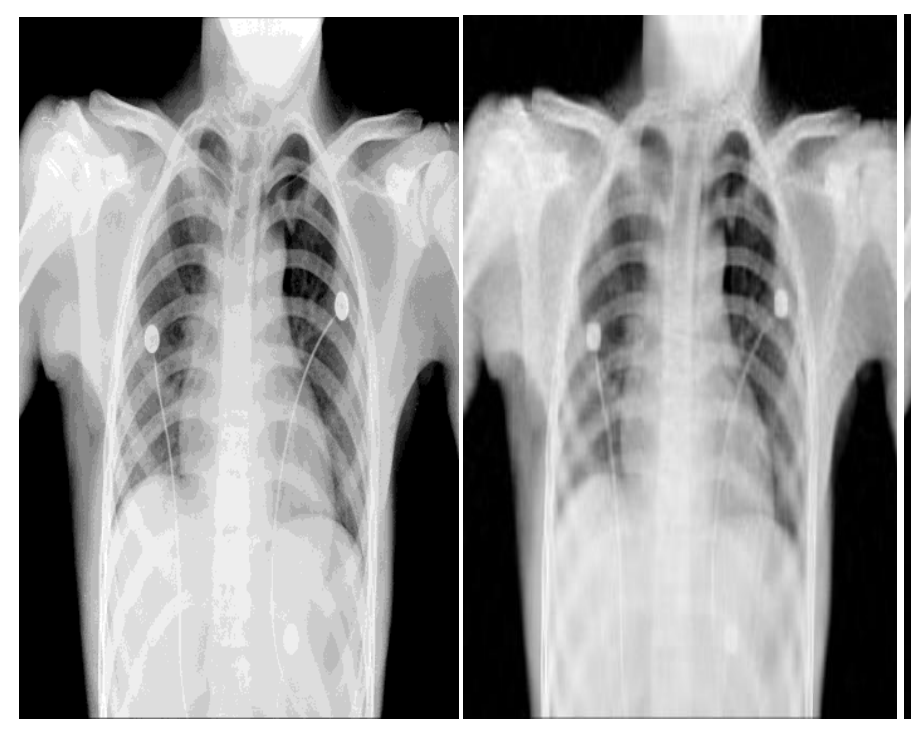

(a) (b)

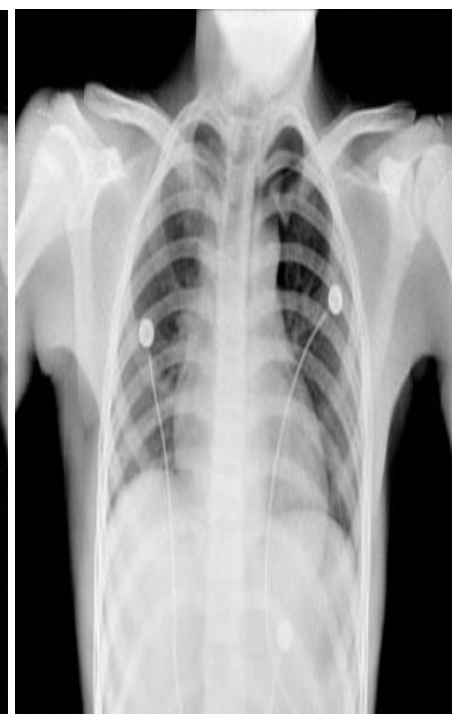

(c)

Figure 4. The Original, Compressed and decompressed images of Chest 

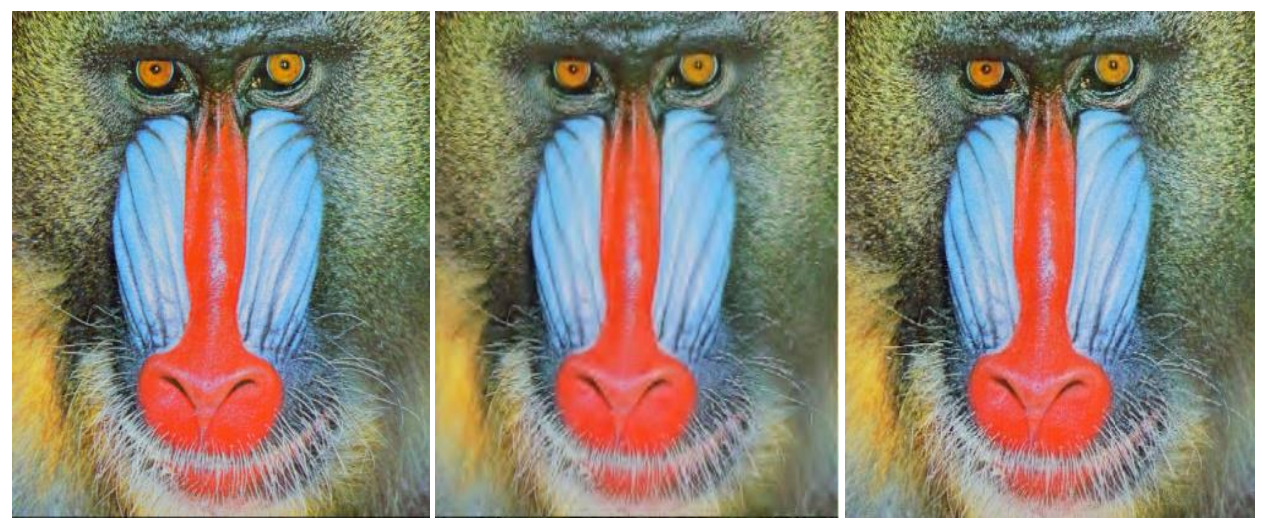

Figure 5. The Original, Compressed and Decompressed Images of Baboon

The work involved in comparing the computation times of various existing algorithms with the proposed algorithm. The results are tabulated in the Table I. which shows the performance of the proposed technique over the existing methods. The comparative graph is shown in the Figure 5.

\section{Table I. Comparison with the Existing Techniques both Compression Efficiency and Computation Time}

\begin{tabular}{|c|c|c|}
\hline Algorithm & $\begin{array}{c}\text { Compression } \\
\text { Efficiency (\%) }\end{array}$ & $\begin{array}{c}\text { Computation } \\
\text { Time(ms) }\end{array}$ \\
\hline Existing 1 & 66 & 121.54 \\
\hline Existing 2 & 55 & 232.36 \\
\hline Existing 3 & 25 & 233.98 \\
\hline Existing 4 & 88.8 & 43.155 \\
\hline $\begin{array}{l}\text { Proposed } \\
\text { Approach }\end{array}$ & $\mathbf{9 2 . 4}$ & $\mathbf{2 1 . 3 7}$ \\
\hline
\end{tabular}

\section{Conclusion}

The developed novel technique for the lossless image compression technique investigated the bottlenecks of the existing algorithms. The novel static sub image based compression proposed with the results. The algorithm performs better from the comparisons and concluded that the proposed work compressed the image along with good quality image reconstruction but also it is effective to reduce the same time is effective in computation time. The algorithm tested on the images of chest and other medical, satellite images and 
standard bench mark images like Aerial, Baboon. This algorithm gives wide scope for further research in the area of lossy image compression as it involves mathematical approach.

\section{Acknowledgements}

The first author feels profoundly indebted to his research supervisor Dr. B.C. Jinaga for the opportunity to work with him and benefit from his valuable guidance and knowledge. The author's special thanks are also due to Dr. I. Gopal Reddy, former Vice-Chancellor of unified J.N.T.University, Hyderabad for his valuable advices and encouragement during his research work.

\section{References}

[1] C. Christopholos, A. Skodras and T. Ebrahimi, "The JPEG 2000 still image coding: An overview”, IEEE transactions on consumer Electronics, vol. 46, (2000), pp. 1103-1127.

[2] M. D. Adams, and F. Kossentini, “ Jasper: A software based JPEG 2000 codes implementation in Proc. of IEEE International Conference of Image processing, vol. 2, (2000), pp. 53-56.

[3] W. Chen and W. Duan, "Computational Aspects of Mathematical Models in Image compression", PhD Dissertation, Department of Communication Engineering, Chalmers University of Technology, G"oteborg, Sweden.

[4] H. Zhang, W.-h. Ai and C.-1. Shen, "MODIS Image compression Based on JPEG 2000" Proc. of $4^{\text {th }}$ International conference on computer science \& Education, (2009), pp. 732-734.

[5] H. Pan, L.-Z. Jin, X.-H. Yuan, S.-Y. Xia and L.-Z. Xia "Context-based embedded image compression using binary wavelet transform”, Image and Vision Computing, vol. 28, no. 6, (2010) June, pp. 991-1002.

[6] E. Delponte, F. Isgro, F. Odone and A. Varri, "SVD matching using SIFT features", Published in Science Direct, Graphical Models, vol. 68, (2006), pp. 415-431.

[7] J. W. Cho, S. Valette, J. H. Park, H. Y. Jung and Prost, "R "3-D mesh sequence compression using waveletbased multi-resolution analysis", Applied Mathematics and Computation, vol. 216, no. 2, (2010) March, pp. 410-425.

[8] H. Liu and S. Ma, "R-D optimized tree-structured compression algorithms with discrete directional wavelet transform", Journal of Computational and Applied Mathematics, vol. 219, no. 1, (2008) September, pp. 302-311.

[9] J. Chen, Y. Zhang and X. Sh, "Image coding based on wavelet transform and uniform scalar dead zone quantizer", Signal Processing: Image Communication, vol. 21, no. 7, (2006) August, pp. 562-572.

[10] S. M. Basha and B. C. Jinaga, "A Novel response dependent image compression algorithm to reduce the nonlinear effects in color images using JPEG", Proc. of IEEE Region 8 International Conference Computational Technologies in Electrical and Electronics Engineering (SIBIRCON), (2010), pp. 122 - 125.

[11] G. Liu, X. Zeng, F. Tian, K. Chaibou and Z. Zheng, "A novel direction adaptive wavelet based image compression", AEU - International Journal of Electronics and Communications, vol. 64, no. 6, (2010) June, pp. 531-539.

[12] B. Usevitech, "Optimal Bit Allocation for Bi-orthogonal Wavelet Coding", Proc. of Data Compression Conference, (1996), pp. 387-395.

[13] L. Lu and S. Deng, "Study on JPEG 2000 Optimized Compression Algorithm for Remote Sensing Image", Proc. Of International Conference on Network Security, Wireless Communications and Trusted Computing, IEEE Computer Society, (2009), pp. 771- 775.

[14] A. H. R. Begum, D. Manimegalain and A. Abudhahir, "Optimum coefficients of Discrete Orthogonal Tchebichef Moment Transform to Improve the Performance of Image Compression", Malaysian Journal of Computer Science, vol. 26, no. 1, (2013), pp. 60-75. 


\section{Authors}

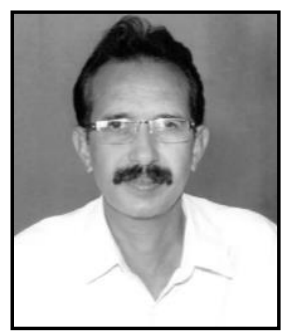

Shaik. Mahaboob Basha, He has done his $\mathrm{PhD}$ from J. N. T. University, Hyderabad. $\mathrm{He}$ is the member of Engineering Professional bodies like IEEE, IE (I), ACM and ISOI and published 15 papers in reputed International and National journals and conferences.Currently,he is Professor and Head of the Department of E.C.E in Priyadarshini College of Engineering and Technology, Nellore.

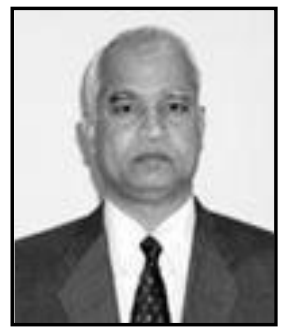

B. C. Jinaga, He has born on June, 1950, graduation from Karnataka University, Dharwad, Post Graduation from Regional Engineering, Warangal and Ph.D., from Indian Institute of Technology, Delhi. He has been with JNT University since 24 years. He is Fellow of The Institution of Engineers (India). He guided several students for Ph.D. He received Best Teacher Award for the year 2002 awarded by Government of Andhra Pradesh. 